\title{
On the ontological status of molecular structure: is it possible to reconcile molecular chemistry with quantum mechanics?
}

\author{
Sebastian Fortin - Martín Labarca - Olimpia Lombardi
}

CONICET - Universidad de Buenos Aires, Argentina

\begin{abstract}
According to classical molecular chemistry, molecules have a structure, that is, they are sets of atoms with a definite arrangements in space and held together by chemical bonds. The concept of molecular structure is central to modern chemical thought given its impressive predictive power. It is also a very useful concept in chemistry education, due to its role in the rationalization and visualization of microscopic phenomena. However, such a concept seems to find no place in the ontology described by quantum mechanics, since it appeals to classical notions such as the position of the atomic nuclei or the individuality of electrons. Although this problem has attracted the attention of several authors, the discussion is far from settled. Some authors adopt an explicitly reductionist position and advocate to reconstruct the concept of molecular structure within the framework of the quantum theory. Others, although acknowledging the conceptual discontinuity between quantum mechanics and molecular chemistry, keep the hope of future reduction alive. From an explicitly non-reductionist position, on the contrary, others authors conceive molecular structure as an emergent phenomenon.

The purpose of this article is to propose a different line of argumentation to address this problem. By contrast to reduction and emergence, the admission of a multiplicity of ontologies, not necessarily linked by hierarchical connections, cancels the need of finding a relation of dependence between the molecular level and the quantum level. This ontologically pluralist position can be applied to the issue of molecular structure, in order to argue that it is possible to admit the existence of structure in the ontology of molecular chemistry, in spite of the fact that it does not exist in the quantum world.
\end{abstract}

\section{Keywords}

Molecular structure, quantum mechanics, reduction, emergence, ontic plurality.

\section{1.- Introduction}

According to classical molecular chemistry, molecules have a structure, that is, they are sets of atoms with a definite arrangements in space and held together by chemical bonds. The concept of molecular structure is central to modern chemical thought given its impressive predictive power. It is also a very useful concept in chemistry education, due to its role in the rationalization and visualization of microscopic phenomena. However, such a concept seems to find no place in the ontology described by quantum mechanics, since it appeals to classical notions such as the position of the atomic nuclei or the individuality of electrons, both ideas strongly challenged in the quantum context. 
Although this problem has attracted the attention of several authors, the discussion is far from settled: the views about the link between quantum concepts and the notion of molecular structure diverge markedly. Some authors adopt an explicitly reductionist position and advocate to reconstruct the concept of molecular structure within the framework of the quantum theory. Others, although acknowledging the conceptual discontinuity between quantum mechanics and molecular chemistry, keep the hope of future reduction alive. From an explicitly non-reductionist position, on the contrary, others authors conceive molecular structure as an emergent phenomenon which, although irreducible to quantum mechanics, ontologically depends on the underlying quantum domain.

The purpose of this article is to propose a different line of argumentation to address this problem. By contrast to reduction and emergence, the admission of a multiplicity of ontologies, not necessarily linked by hierarchical connections, cancels the need of finding a relation of dependence between the molecular level and the quantum level. This ontologically pluralist position is not new in the discussions about the relationships between chemistry and physics. Sometimes rooted in a philosophically Kantian framework, ontological pluralism was appealed to in order to defend the ontological autonomy of chemistry and the objective existence of orbitals. In this case, the aim is to apply that pluralist position to the issue of molecular structure, in order to argue that it is possible to admit the existence of structure in the ontology of molecular chemistry, in spite of the fact that it does not exist in the quantum world.

\section{2.- Why reduction?}

The idea of ontic reduction has a venerable tradition both in the history of philosophy and in the history of science. Already in Pre-Socratic philosophy, the idea of a fundamental stuff which everything is made of was the trademark of the Milesian school in its search for reducing multiplicity to unity. Although this initial monism was later replaced by an ontic picture based on several material principles, the attempt to reduce the diversified empirical reality to a simpler underlying realm survived in Empedocles and his four elements and in the atomism of Leucippus and Democritus. In Plato's philosophy, in turn, the principles became non-material. Platonic Ideas retained ontological priority over the other ontic items: Ideas did not need anything else to exist; the remaining items had a secondary existence since they required the primary items to exist -sensible things were mere "copies" of Ideas.

In the Modern Age, the notion of ontic reduction reappeared in two senses. On the one hand, the relationship between primary qualities, endowed with ontological priority, and merely subjective secondary qualities permeated the philosophy of Locke and the physics of Galileo. On the other hand, Ancient atomism, first introduced in Modern Europe by Gassendi, was reborn in Boyle's corpuscular philosophy, which, in turn, strongly influenced later 
physics, such as Newton's corpuscular theory of light. The influence of Ancient atomism arrived to the nineteenth century through John Dalton's modern atomic theory.

Still closer to our times, two of the most famous physicists of the late nineteenth century conceived their fields of study in reductive terms. Under the assumption that gases are nothing else than particles in mechanical interaction, Boltzmann tried to explain thermal phenomena in gases in terms of classical mechanics. Meanwhile, Maxwell devoted much of his scientific effort to the reduction of electromagnetic phenomena to mechanical vibrations of a luminiferous aether. In both cases, the underlying ontological assumption was that Nature is made of mechanical entities governed by Newtonian physics; this assumption was precisely what justified the strategies directed to explain the new theories (thermodynamics, electromagnetism) by means of classical mechanics. Nowadays, ontic reductionist ideas are still ingrained in many areas of science. Perhaps the most striking example is the present-day particle physics as embodied in the Standard Model, which supposedly describe those tiny elemental entities which the entire reality is composed of.

Given the long tradition history of the idea of ontic reduction, it is not surprising that it also entered the field chemistry. The reductionist stance was stimulated by the optimism following the success of quantum mechanics, as expressed by Paul Dirac's famous claim about the explanation of "the whole of chemistry" in quantum terms (Dirac 1929: 714). In this way, chemistry turns out to be part of the ontic interdisciplinary hierarchy favored by the reductionist view of nature, according to which "chemistry tells us that a piece of wood is 'really' a complicated arrangement of many kinds of molecules bound together; atomic physics tells us that molecules are 'really' various atoms held together by interatomic forces; particle theory tells us that atoms are 'really' elementary particles in interaction, and so on" (Rohrlich 1988: 295-296). Once the assumption of reduction is admitted, it directly affects the concept of molecular structure, since it is not an auxiliary or secondary notion, but a central concept of chemistry: molecular structure is "the central dogma of molecular science" (Woolley 1978: 1074). As Robin Hendry claims, "molecular structure is so central to chemical explanation that to explain molecular structure is pretty much to explain the whole of chemistry" (Hendry 2010: 183).

A representative of an explicitly reductionist stance is Hinne Hettema (2012), who considers that molecular chemistry can be reduced to quantum mechanics when the traditional definition of reduction is adequately adjusted. According to him, the fail to recognize reduction is due to, at least partially, the scarce impact of the recent developments in quantum chemistry on the present-day philosophy of chemistry. However, when the relationships between molecular chemistry and quantum mechanics are analyzed in detail, Hettema admits that the links do not supply a global reduction but only local and partial reductions of particular theories of chemistry; they introduce relevant idealizations and approximations that 
establish loose and non-continuous connections between theories; they even draw concepts out of context and re-use them in a manner inadmissible to the theory to which the concepts originally belong. In brief, those links supply a liberal notion of reduction, which could even be made compatible with non-reductionist positions, so liberal that one is entitled to ask why the relationship is still called 'reduction' instead of 'inter-theory link' (for a detailed criticism, see Lombardi 2014a).

Another reductionist strategy is that relying on the concept of quantum decoherence: conceived as the process that accounts for the classical limit of quantum mechanics (Zurek 1991, 2003), environment induced decoherence would supply the necessary connection between the classical concepts of molecular chemistry and the quantum domain (Trost and Hornberger 2009). For example, when discussing the problem of optical isomerism, Eric Scerri points out that "this problem has gradually begun to dissolve with the growing realization of the role of quantum decoherence in physics and other disciplines." (Scerri 2011: 4; for a similar claim, see Scerri 2013). However, in the domain of the philosophy of physics, a deep skepticism about the relevance of decoherence to the emergence of classicality prevails. As Guido Bacciagaluppi (2012) stresses in his article about the role of decoherence in quantum mechanics, although naive claims of the kind that decoherence gives a complete answer to the measurement problem are still somewhat part of the "folklore" of the matter, decoherence as such provides a solution neither to the measurement problem nor, in general, to the classical limit problem, at least not unless it is combined with an appropriate interpretation of the theory (for specific arguments, see Fortin, Lombardi and Martínez González 2016).

At present, the strongest reductionist strategy regarding molecular structure is that represented by the so-called Quantum Theory of Atoms in Molecules (QTAIM), proposed by Richar Bader in the decade of 1990's (Bader 1991, 1994). According to the theory, molecular structure can be obtained from the topological properties of the electron density distribution function of the system, which is defined in the physical three-dimensional space. The strategy consists in computing the zero flux surfaces in the gradient vector field of the electron density: the closed two-dimensional zero-flux surfaces divide the molecular three-dimensional space into separate mononuclear regions called 'atomic basins'. On the other hand, the positions of the atomic nuclei are identified with relative maximums of the electron density. Then, an atom in a molecule is defined as the union of a nucleus and its associated basin. In turn, given two atoms, they are bonded if a single line of locally maximum density in threedimensional space, termed 'bond path' and linking their nuclei, can be identified: the existence of a bond path is a universal indicator of chemical bonding. The QTAIM had a strong impact in the quantum chemistry community, since it supplied a visually appealing picture of a molecule and its structure. This led some authors, such as Hinne Hettema (2012), to share Bader's optimism about his reductionist program: "The time has arrived for a sea 
change in our attempts to predict and classify the observations of chemistry, time to replace the use of simplified and arbitrary models with the full predictive power of physics, as applied to an atom in a molecule." (Bader 2011: 253). However, optimism should be moderated by two essential features of the theory. First, as any approach in quantum chemistry, the QTAIM relies on the assumption of the Born-Oppenheimer approximation (BOA): from being originally considered an innocent approximation, in the last times the BOA has been reconceptualized as a substantial addition to quantum mechanics. In fact, the BOA introduces the molecular structure into the quantum description from the very beginning, when the positions of the nuclei are established with the appeal to classical geometric considerations. On the other hand, the assumption of the nuclei at rest in fixed spatial positions is in contradiction with the Heisenberg principle, which prevents quantum systems from having definite values of position and velocity simultaneously (for a detailed argumentation, see Lombardi and Castagnino 2010). Second, the result of using the electronic density for each electron of the system instead of the many-electron wave function is a crude approximation: the QTAIM works with a fictitious system of independent electrons which has the same ground-state density as the quantum molecule, but where all the interactions between electrons have been cancelled.

Although admitting the impossibility of deriving molecular structure from quantum mechanics, some authors with reductionist spirit consider that this impossibility is not the result of a conceptual obstacle. By contrast, it would be the consequence of our partial knowledge of the molecular systems in the theoretical framework of quantum mechanics, a limitation that will gradually be overcome in the future. This is the position of Guy Woolley and Brian Sutcliffe, when they say: "We have never claimed that molecular structure cannot be reconciled with or reduced to quantum mechanics, or that there is something 'alien' about it; our claim is much more modest. We do not know how to make the connection." (Sutcliffe and Woolley 2011: 94; see also Sutcliffe and Woolley 2012).

In spite of the prevailing reductionist perspective in the scientific community, during the last decades some voices rise to point out the shortcomings of the assumption of reduction in the field of chemistry. One of the first authors in stressing those shortcomings was Hans Primas, who identified non-locality as the specific feature of quantum mechanics that excludes the spatial concept of molecular structure: "the holistic correlations between the nuclei and electrons are suppressed, so the description of a molecule reduces to the description of the motion in the electrical field of a classical nuclear framework" (Primas 1998: 91; see also Primas 1983). In a similar vein, Anton Amann recalls that, in order to conceive the molecule as an individual object with its own spatial structure, it is necessary to ignore quantum correlations: "The shape of a molecular state should of course not show holistic correlations to other molecular quantities and hence be unambiguously defined" (Amann 1992: 32). In turn, if the state of the molecule determines its properties, the 
possibility of superpositions has to be dismissed; for instance, in the case of chemical isomers: "What is the shape of the hypothetical superposition of these two species? Is there a proper reason to exclude such superpositions? What reason can be given from a quantummechanical point of view-for chemical systematics?" (Amann 1992: 32).

In fact, the case of optical isomerism is perhaps the clearest challenge to the assumption of reduction. As Wooley himself recognizes, "the existence of isomers, and the very idea of molecular structure that rationalizes it, remains a central problem for chemical physics." (Woolley 1998: 3). The so-called Hund's paradox reads: since chiral states -corresponding to levo-rotation and dextro-rotation- are not eigenstates of the Hamiltonian -which is invariant under spatial reflection- and none of them corresponds to the basal state, why do certain chiral molecules display an optical activity that is stable in time, associated to a well-defined chiral state?, why are they not in a superposition of the two possible chiral states? (Hund 1927). More recently, the paradox was formulated in a slightly stronger version (Berlin, Burin and Goldanskii 1996): why do chiral molecules have a definite chirality?

The inability of quantum mechanics to explain optical isomerism is a particular case of what can be called, following Woolley and Sutcliffe (1977), the 'symmetry problem': if the interactions embodied in the Hamiltonian of the molecule are Coulombic, the solutions of the Schrödinger equation are spherically symmetrical; however, the asymmetry of polyatomic molecules is essential in the explanation of their chemical behavior. On this basis, Robin Hendry concludes that "if the acidic behaviour of the hydrogen chloride molecule is conferred by its asymmetry, and the asymmetry is not conferred by the molecule's physical basis according to physical laws, then surely there is a prima facie argument that ontological reduction fails." (Hendry 2010: 186).

The above arguments mainly come from chemists and philosophers of chemistry, who express the difficulties found in the scientific practice to supply quantum explanations to chemical phenomena. But strong arguments against the idea of reducing molecular chemistry to quantum mechanics come also from physics: the reasons come from the very theoretical structure of quantum mechanics.

In 1967, Simon Kochen and Ernst Specker (1967) presented a fundamental theorem that proves, in the context of the Hilbert space formalism, that any assignment of a definite value to all the observables of a quantum system leads to contradiction. This means that quantum mechanics is essentially contextual: definite values can be consistently assigned only in a context, that is, to observables that share the same eigenbasis. It is essential to stress that it is not the case that the quantum system has definite properties but we do not know which; on the contrary, any non-contextual attempt to complete the assignment of properties, no matter how, leads to contradiction. It is not the case that the particle has a definite momentum but we do not know its position: any assignment of a definite position and a definite position is logically 
forbidden by the very structure of the theory. Therefore, quantum mechanics challenges the traditional principle of omnimode determination, "quodlibet existens est omnimode determinatum", that appears in the works of Christian Wolff (1728), in the famous treatise on the calculus of probabilities by Jakob Bernoulli (1713), and is also repeated several times by Immanuel Kant in his lectures on metaphysics dating from the 1760s to the 1790s (see the edition of 1902). A quantum system is not an individual in the traditional sense, since it has properties that have no definite value; and this is not a merely epistemic limitation, but an ontological fact described by the theory.

Another peculiarity of quantum mechanics is the fact that the statistical behavior of quantum "particles" does not follow the rules of classical particles: whereas classical particles follow the Maxwell-Boltzmann statistics, their quantum counterparts follow Fermi-Dirac or Bose-Einstein statistics, which also show that they do not behave as individuals in the traditional sense. The category of individual requires some "principle of individuality" that makes an individual different from other individuals and that re-identifies it through time (French and Krause 2006). Quantum "particles", by contrast, do not preserve their identity when statistically conceived, they are indistinguishable, and this does not depend on the complexity of the system, but on the very nature of quantum mechanics. Paul Teller (1998) addresses the problem of indistinguishability in terms of the concept of haecceity, as introduced by John Duns Scotus in the thirtheenth century: haecceity is what makes an individual to be different from all others in some way that trascends all properties. According to Teller, quantum mechanics provides good reasons for rejecting any aspect of quantum entities that might be thought to do the job of haecceity and, then, support their individuality: "I suggest that belief in haecceities, if only tacit and unacknowledged, plays a crucial role in the felt puzzles about quantum statistics" (Teller 1998: 122).

The features of contextuality and indistinguishability specific of quantum mechanics are clear obstacles to define molecular structure in terms of the spatial relations of the nuclei conceived as individual localized objects. According to quantum mechanics, quantum "particles" are not individuals with definite properties, which can be identified as different from others and re-identified through time; therefore, they do not have the ontological stability necessary to maintain the relations that can lead to the identifiable shape of a spatially definite system, as molecular structure requires.

Another aspect of molecular chemistry, when expressed in quantum terms, is the priority conferred to the Hamiltonian of the molecule. As it is well known, a quantum system is represented by a Hilbert space, where all the bases are equivalent: there is no theoretical reason to prefer a basis over the others. However, chemistry always works in the basis of the energy: the interest is always focused on the possible values of the energy and the possible eigenstates of the Hamiltonian. Another way of stressing this point is to notice that, in 
chemistry, it is usual to talk about the time-independent Schrödinger equation; so the Hamiltonian seems to have a privilege since contained in the law to be solved. Nevertheless, in the context of quantum mechanics, the Schrödinger equation is the dynamical postulate of the theory, that is, the law that rules the time evolution of the system. Therefore, talking of a dynamical postulate that is 'independent of time' sounds, at least, strange from a physical viewpoint. Strictly speaking, the so-called 'time-independent Schrödinger equation' should be conceived as the eigenstate-eigenvalue equation of the Hamiltonian. But, from this perspective, it turns out to be clear that one can solve the eigenstate-eigenvalue equation of any observable of the system. So, again, the privilege conferred to the Hamiltonian needs to be justified. Forgetting this may lead to some reconstructions of the theory that restrict the space of observables to a single observable, the Hamiltonian (see Hettema 2012: 264-265), depriving quantum mechanics from its specificity, strongly related to the existence of incompatible observables. A possible answer to this requirement of justification is that chemists are mainly interested in the stationary states of the molecule (eigenstates of the Hamiltonian) and in the energy of the molecule in those states. But this assumes that energy is always a definite-valued property. However, this is not an innocent assumption since, as stressed above, the Kochen-Specker theorem teaches us that not all the observables of the system may have definite values simultaneously. From this perspective, the need for the justification of the privilege of energy takes the form of the need for the justification of the definite-valuedness of the Hamiltonian. In summary, although quantum chemists use the resources supplied by quantum mechanics with successful results, this does not mean reduction: their applications add certain assumptions not justified in the context of quantum mechanics or even inconsistent with the very formal structure of the theory.

\section{3.- Why emergence?}

By contrast to the notion of reduction, with its venerable and long tradition, the idea of emergence has a relatively recent appearance in the history of thought. At the beginning of the twentieth century, the so-called 'British emergentists' were the first to attempt to understand certain scientific phenomena by appealing neither to mechanicism nor to vitalism. From the perspective of those first emergentists, most mental, biological and chemical properties and processes must be conceived as emergent. However, the rest of the twentieth century, under the strong influence of logical positivism, was marked by reductionism. It was only during the last decades that the notion of emergence has "reemerged" not only in philosophy, but also in the scientific field (Cunningham 2001). The reasons for this revival are varied. One of them is the high development of the interdisciplinary field of the sciences of complexity. Another reason can be found in the studies about mind and consciousness. Of course, the boom in the computational resources to model non-linear dynamical systems has also exerted a great influence on the appeal to the notion of emergence in different scientific disciplines. In the 
field of philosophy, the return of emergentism was possible thanks to the collapse of logical positivism, along with its search for the reductive unification of sciences.

The main difficulty in the discussions about emergence is the fact that there are as many meanings of the word 'emergence' as authors that use it. The general notion of emergence is very appealing: in relation to the entities from which they arise, emergents are usually characterized as novel, unpredictable, unexplainable on the basis of a lower level domain. In Philip Anderson's terms, the whole is not merely greater than but essentially different from the sum of the parts (Anderson 1972). But as soon as one tries to make the general idea more precise, multiple views arise, varying from one author to another. In this sense, Jaegwon Kim claims: "The term 'emergence' seems to have a special appeal for many people; it has an uplifting, expansive ring to it, unlike 'reduction' which sounds constrictive and overbearing. We now see the term being freely bandied about, especially by some scientists and science writers, with little visible regard for whether its use is underpinned by a consistent, tolerably unified, and shared meaning" (Kim 2006: 547). For this reason, it is convenient to begin by introducing certain terminological precisions.

A first distinction is that between diachronic and synchronic emergence (Rueger 2000, Humphreys 2008). Diachronic emergence is a time process through which a novel item arises from a pre-existent domain: the emergent item is genuinely new since it comes into existence at a certain time as the result of previous events. Synchronic emergence, by contrast, refers to the relationship between a certain item and a lower level: the emergent item arises out of an underlying domain, but is neither reducible nor predictable from that domain. It is clear that, in the problem of the relations between chemistry and physics, synchronic emergence is the relevant concept. Another distinction to be taken into account is that between epistemic and ontic emergence (see O'Connor and Wong 2015). Epistemic emergence depends on the limitation of human knowledge: it is that limitation what makes the emergent item neither explainable nor predictable from the basal level. Ontic emergence, by contrast, refers to the relationship between items belonging to different ontic levels, sometimes expressed in mereological terms: the emergent items, which arise from a lower level, are ontically new and populate reality as objectively as the items belonging to the lower level.

Robin Hendry (2004, 2008, 2010), who has largely treated the issue of molecular structure, clearly distinguishes between the intertheoretic-epistemic and the metaphysicalontological aspects of the reduction debate. In fact, reductionists and non-reductionists agree in that classical intertheoretic reductions of chemistry are not currently available, but differ in how they interpret the situation: "the issue is essentially future directed -both sides must wait and see, even if they would bet different ways. But why do the two sides make different bets? Perhaps the answer concerns their different underlying metaphysical views." (Hendry 2010: 184). On this basis, Hendry correctly claims that the debate chemistry-physics must turn to 
consider the ontic relationships between the entities, processes, and laws studied by the two different sciences. In particular, the relationship between quantum mechanics and molecular chemistry, embodied in the way in which the molecular structure of a system of several electrons and nuclei is explained, must be considered in terms of emergence, conceived as an ontological concept that refers to an ontic relation.

One might suppose that, once the focus is put on synchronic ontological emergence, the misunderstandings have been overcome. However, this is not the case: there are disagreements about what ontological categories - objects, properties, processes, etc. - may be emergent; sometimes emergence is identified with other notions, such as supervenience; there is no consensus about whether downward causation is a requiriement for emergence or not; etc. This is not the place to discuss all these matters. Nevertheless, despite the many disagreements, there are several features shared by almost every emergentist position. Virtually all emergentists hold a hierarchical view, according to which reality is organized in different ontic levels or strata. In turn, emergence is traditionally conceived as the symptom of a failure of reduction. Moreover, emergence is an essentially asymmetric relation: if $A$ emerges from $B$, then $B$ does not emerge from $A$. Usually, it is said that emergent items have novel causal powers, namely, that they are capable of producing genuinely novel effects in their own level. If one prefers to avoid the talk about causal powers, it might be said that emergents are actively involved in new regularities in their ontic domain.

Most emergentists base the asymmetry of emergence on physicalism: the belief that there are no existent items beyond physical items, and that all real regularities depend on the fundamental regularities of the basal domain of physics. This assumption endows the physical level with ontic priority over the remaining levels of reality, and this naturally supports the theoretical priority of physics over other special sciences. However, physicalist emergentists face a conceptual problem: their view seems to contradict the metaphysical principle that something cannot come from nothing (O'Connor 1994). In fact, if all the items belonging to the non-basal levels are realized by physical items, how can genuine novelty emerge? As Alexandru Manafu clearly explains in the particular case of chemical items: "It is hard to miss the apparent tension between the two claims made in the preceding paragraphs. If chemical stuff is composed of nothing else except micro-physical stuff, how can one justify the belief that there are even such things as chemical properties, truths, or explanations as opposed to merely complex quantum-mechanical properties, truths, or explanations? Given the generality of physics, how can the autonomy of chemistry be preserved in a substantial way, i.e., how can chemistry be considered autonomous in a way which goes beyond historical or methodological autonomy? If physicalism is true, can we even speak of the ontological autonomy of chemistry, as opposed to a merely historical or methodological autonomy?" (Manafu 2011: 10). 
Setting aside for a moment the above difficulty, let us consider the asymmetry essential to emergence: this asymmetry means that emergence does not involve mere correlations, but requires something else, in particular, ontic dependence: if $B$ emerges from $A, B$ ontically depends on $A$, or, in other words, $A$ has ontic priority over $B$. The ontic dependence implicit in emergence is usually expressed in counterfactual terms, such as 'if $A \operatorname{did}$ not exist, then $B$ would not exist either.' The relevant question regarding the decision about accepting or rejecting emergentism is: why to accept the ontic dependence of the emergent level on the basal domain? What argument does support the ontic priority of the basal domain over the emergent level? Of course, there is no way to decide the truth value of a counterfactual proposition beyond any doubt: we always rely on indirect arguments to justify our commitment to an ontological thesis like that. In this case, two types of arguments can be considered: conceptual and pragmatic.

The conceptual argument appeals to the scientific reason we have to believe in the existence of the basal and the emergent levels: in the field of science, we accept the items objects, properties, processes- described by our best theories. In particular, our belief in the existence of the subatomic systems described by quantum physics and of the molecules described by molecular chemistry are supported by the great success of the respective theories. Therefore, we can assess the ontological counterfactual 'if $A$ did not exist, then $B$ would not exist either' on the basis of the acceptability of its epistemological counterpart: 'if the theory describing $A$ were wrong, then the theory describing $A$ would also be wrong.' This means that we must ask ourselves to what extent the knowledge derived from the theory of the emergent level depends on what the basal theory offers: if we did not have access to the resources supplied by the theory that describes the ontically prior level, would the knowledge of the supposedly dependent level be affected? The answer to this question does no longer belong to the ontological field, but refers to what effectively happens in science, and its truth value depends on the particular relationships between the two theories. In the particular case of the quantum reduction of molecular structure, the question is: would we lose the chemical knowledge about molecules if we did not count with the resources supplied by quantum mechanics? As Jaap van Brakel explicitly asserts, the answer of this question is mostly negative: "If quantum mechanics would turn out to be wrong, it would not affect all (or even any) chemical knowledge about molecules (bonding, structure, valence and so on). If molecular chemistry were to turn out to be wrong, it wouldn't disqualify all (or even any) knowledge about, say, water" (van Brakel 2000: 177). This means that the dependence relation between theories does not offer good conceptual arguments for support the ontic dependence of molecular structure on the quantum domain.

The issue of the ontic priority of quantum mechanics over molecular chemistry can also be analyzed from a pragmatic perspective, by considering the pragmatic virtues of the two theories regarding to what Ian Hacking (1983) calls the intervention on reality. As this author 
claims, in the discussions about the foundations of science we have paid too much attention to theoretical considerations, forgetting the effective practice of science: it is in this pragmatic context that the criterion for the existence of scientific entities has to be searched for. According to Hacking, we accept the existence of unobservable entities when we can "spray them", that is, when we can use them for intervening in other aspects of nature: "We are completely convinced of the reality of electrons when we set out to build -and often enough succeed in building-new kinds of devices that use various well-understood causal properties of electrons to interfere in other more hypothetical parts of nature" (Hacking 1983: 265). In other words, it is scientific experimental practice, and not descriptive matters about theories, which gives us the best support for our commitments about scientific reality. But if this is the case, molecular chemistry offers an excellent example of ontic independence. The practice of chemistry shows a wide scientific field where it is possible to develop a highly fruitful work on the basis of the concept of molecular structure, in spite of the fact that it cannot be reduced to quantum mechanics. From this pragmatic viewpoint, "molecular chemistry holds the winning card: its astonishing success in the manipulation of known substances and in the production of new substances is the best reason for accepting the existence of the entities populating its realm. In other words, we are entitled to admit the reality of the molecular world -inhabited by, among others, chemical orbitals, bonding, chirality, molecular shapeson the basis of the impressive fruitfulness of molecular chemistry itself, independently of what physics has to say about that matter." (Lombardi and Labarca 2011: 74). As a consequence, not only the theoretical virtues of chemistry, but primarily its pragmatic virtues are the factors that play a decisive role in the arguments for the autonomy of the chemical domain and against the assumption of ontic dependence (see also Lombardi 2014b).

Summing up, the crisis of the traditional idea of the reduction of chemistry to physics led to the adoption of emergence as a non-reductionist perspective that would overcome the difficulties of the reductive view. Nevertheless, emergentism incorporates metaphysical assumptions about the ontic dependence of the molecular domain on the quantum realm that, far from being scientifically supported, seem to re-edit the traditional hierarchy of sciences supported by positivism.

\section{4.- Ontic plurality: Why not?}

Why is so much ink spilled on the problem of molecular structure? Because the debate focuses not on an auxiliary or secondary notion, but on a central concept of molecular chemistry: molecular structure is "the central dogma of molecular science" (Woolley 1978: 1074). More precisely, "[t]he alpha and omega of molecular chemistry is the doctrine that molecules exist as individual objects and that every molecule has a shape, characterized by its molecular frame" (Primas 1994: 216). In fact, the structure of a molecule plays a central role in the explanation of the chemical properties of the corresponding substance: solubility, 
differences in melting and boiling points, reactivity, acidity, density, polarity, are some of the many properties in whose explanation the concept of molecular structure plays an essential role. Moreover, geometrical structure is extremely helpful to visualize the object of study of molecular chemistry: it is very difficult to imagine a molecule without its geometrical shape in three-dimensional space. Additionally, structure is a powerful tool to interpret the result of experiments. For example, when we see the result of the imaging of the molecule of pentaceno by means of the atomic force microscopy (AFM) technique (see Gross et al. 2009, Figure 1), it is difficult to not identify the spatial disposition of the molecule's atoms in the AFM picture.

It is also very important to emphasize that the concept of molecular structure is not an isolated concept, whose reduction to quantum mechanics would merely simplify our picture of reality. Molecular chemistry is not a "zoo" of structures that only deserve to be classified by geometrical similarities; on the contrary, it is a scientific discipline that involves a body of theoretical knowledge that allows chemists to make predictions of chemical properties and to operate successfully on reality (we thank Joachim Schummer for drawing our attention to this point, see Schummer 1998). Therefore, the existence of the entities, properties and processes described by that discipline should be assessed in terms of the success of that very discipline and not by means of criteria coming from another disciplinary field.

On the other hand, in spite of its name, molecular chemistry also includes the study of substances, like water, metals, and salts, which obviously evade molecular approaches. In turn, structure is not only related to molecules (we thank Guillermo Restrepo for drawing our attention to this point): metals and other crystalline substances have structure even if they are not composed of molecules; and their structure, given by the spatial relations of their atoms, is also essential to their properties and behaviors. This means that there is a wide domain of entities, properties and processes whose ontic status is under discussion due to their relations to the supposedly underlying quantum domain. As a consequence, when the assumptions of reductionism and of emergentism applied to this case manifest their limitations, it is worth considering a third philosophical position: ontic pluralism.

The idea of a plurality of scientific words is not new in the philosophy of science. On the basis of his semantic conception of reference, Willard V. O. Quine argues that there is no absolute reality: his dictum "To be is to be the value of a bound variable" (Quine 1948) implies that different theories have different ontic commitments. Therefore, "all ascription of reality must come rather from within one's theory of the world; it is incoherent otherwise" (Quine 1981: 21). In the so-called 'historicist turn', it is not difficult to find the idea of conceptual relativity that leads to an ontic pluralism. For instance, according to Thomas Kuhn (1990), the taxonomic categories used by a speaker of a certain language not only describe the 
world in a certain way, but also constitute the ontic items that populate that world; therefore, speakers of different languages may live in different worlds.

A very travelled road toward ontic pluralism is that based on transcendental philosophy. According to Kant, there exists a reality that is independent of the knowing subject: but it is a noumenal realm, which is not object of our knowledge. Speculating about an independent ontic domain makes no sense, since there are no ontic items -objects, properties, processesin the noumenal reality: the noumenon is ineffable, it can only be conceived as a limit of thought. The world, inhabited by the objects of knowledge, results from the synthesis between the categories of the understanding and the material coming from the experience: thoughts without content are empty, intuitions without concepts are blind (Kant 1781: A51, B75; in the original: "Gedanken ohne Inhalt sind leer, Anschauungen ohne Begriffe sind blind"). Therefore, the world our scientific knowledge refers to is not a mere "epistemologized" ontology resulting from our contingent means of access to reality, in the same sense as the Kantian system is not an epistemology but a broad philosophical framework that establishes the necessary conditions of possibility for knowledge and, therefore, for any meaningful scientific discourse.

After having adopted a precritical realism for years, in his book Reason, Truth and History Hilary Putnam defends an explicitly Kantian rooted "internalism", also referred to as "God's Eye point of view" (Putnam 1981: 49), according to which the world exists independently of our theories and consists of some fixed totality of self-subsistent objects. According to Kantian-inspired internalism, on the contrary, "objects' do not exist independently of conceptual schemes. We cut up the world into objects when we introduce one or another scheme of description." (Putnam 1981: 52). In other words, objects depend on conceptual schemes in a strong sense, which includes existence. Therefore, even though there is a reality independent of the subject -a noumenal reality-, the structure of our world only arises from a conceptual scheme. The ontic items resulting from the synthesis between each conceptual scheme and the noumenal reality are the only inhabitants of our ontic domain. In turn, recognizing that different conceptual schemes may coexist leads to the thesis of ontological pluralism, according to which each successful conceptual scheme constitutes its own ontic realm.

Another author directly influenced by Kant's philosophy is Roberto Torretti, who explicitly criticizes metaphysical realists when they "speak persistently of the reality of the external world, as if they were disembodied spirits contemplating it from the outside, and, for all their godlessness, they put forward a view of it that is only conceivable from the standpoint of an omniscient God." (Torretti 2000: 114). Torretti is interested in recovering the elements of Kantian views that are fruitful for the contemporary philosophy of science. So, he admits that Kant's "conception of the understanding as a closed system of rules with full 
authority over every cognitive judgment makes it virtually impossible to think that there is more than one form of understanding, expressed in a single fixed «categorial framework» or «conceptual scheme»" (Torretti 2008: 87). Nevertheless, once it is accepted that there is no reason why the constitution of the ontic domains of our sciences must be binded by a rigid and absolute system of categories, then it is possible to admit that "Kant opens a wide door to intellectual pluralism." (Torretti 2008: 87).

It is interesting to notice that ontic pluralism has begun to enter also the field of the philosophy of biology, when the ontic status of certain central biological concepts is discussed. For instance, John Dupré's "promiscuous realism" can be conceived from an ontic pluralist perspective (Dupré 1993): according to the author, contemporary scientific understanding of biological species lends little support to essentialism and, as a consequence, a variety of more or less cross-cutting classificatory systems is needed (Dupré 2000).

In the field of the philosophy of chemistry, an explicitly Kantian-rooted ontic pluralism has been adopted by Olimpia Lombardi and Martín Labarca $(2005,2006)$ to defend the ontological autonomy of the chemical world with respect to the world of physics. Once it is realized that different theories -and even different disciplines- are accepted at the same historical time due to their pragmatic success, it turns out to be clear that different ontic realms may coexist to the extent that each one of them is constituted by its corresponding conceptual scheme. But since the privileged viewpoint of "God's eye" does not exist, there is not a single "true world" to which all the descriptions must refer: all the ontic domains coming from successful science have the same status, since all of them are constituted by equally objective descriptions. It is from this viewpoint that the multiple and different scientific practices become intelligible.

This ontic pluralism based on Kantian philosophy has been recently applied to different problems in the philosophy of chemistry. In particular, it has been appealed to discussing the problem of the existence of orbitals (Labarca and Lombardi 2010, Lombardi and Labarca 2011): "whereas in quantum mechanics 'orbital' is a non-referring term, in molecular chemistry orbitals exist as spatial regions on the basis of which the shape of the local and individual molecules can be explained" (Labarca and Lombardi 2010: 155). After stressing the conceptual discontinuity between molecular chemistry and quantum mechanics, it is argued that the transition from the concept of orbital as a wavefunction to the concept of orbital as a spatial region of high electronic density is not a continuous transformation within quantum mechanics, but a conceptual breakdown with respect to the supposedly "fundamental" theory. In the context of the debate about natural kinds in chemistry, Mariana Córdoba and Lombardi (2013) notice that, surprisingly, in the attempts to avoid both the Scylla of conventionalism and the Charybdis of essentialism, Kantian philosophy is usually not even mentioned: the debate proceeds from an "externalist" metaphysical perspective, as if 
we could adopt God's eye position to decide what exists and what does not exist in the world. Ontic pluralism allows the authors to argue that, although chemical kinds are not conventional groupings with no reference in the world, they neither are essential and absolute kinds that populate reality in itself. The active role played by the subject in knowledge is what supports the fact that natural kinds result from the synthesis between the noumenal reality and a conceptual framework expressed by a discourse that is the verbal manifestation of a successful scientific practice. More recently, this Kantian-rooted ontic pluralism has been further articulated with the purpose of answering the criticisms advanced from different perspectives since its original formulation (Lombardi 2014b).

When the discussions developed in the previous sections are now reconsidered in the light of ontic pluralism, it is clear that this philosophical framework can also be directly applied to the problem of the ontological status of molecular structure. Furthermore, the arguments against reduction and emergence in this context turn out to become arguments in favor of ontic pluralism.

On the one hand, if the structure of molecules could be explained in terms of quantum mechanics, there would be a good argument in favor of ontic reduction. But as made clear in Section 2, when the relation between molecular chemistry and quantum mechanics is studied in detail, it can be concluded that there are no direct reductive links that would deprive the concept of molecular structure of its own ontic reference. And this conclusion does not depend on approximations on the quantum computations, whose remotion would finally discover the real nature of chemical molecules: even if a quantum omniscient demon gave us the completely precise wave function of the molecule, as the solution of the Schrödinger equation including completely all the subatomic particles and its interactions, it would be impossible to recover, from that solution, the chemical concepts that molecular chemistry uses for its scientifically successful development, such as bonding, functional groups, interatomic distances and angles, and so on.

On the other hand, it is precisely that successful development, founded on its own concepts and methods, what counts for the pragmatic independence of molecular chemistry with respect of quantum mechanics and, with it, what undermines the assumption of an ontic dependence of molecular structure on the quantum domain. This does not mean to ignore the use of certain quantum resources in the context of molecular chemistry; but they are precisely mere auxiliary resources, computational tools always subordinated to the specifically chemical conceptual framework. Therefore, once it is accepted that there are no sufficient arguments to support ontic dependence, the idea that molecular structure is an emergent property that arises from underlying quantum entities loses its essential component.

Ontic pluralism provides a philosophical framework to accommodate these intetheoretic facts. Pluralism stands against the asymmetry presupposed by reductionism and implicit in 
emergentism. Given that the supposedly reduced/emergent domain turns out to be as constituted as the supposedly basal domain, and since there is no external perspective from which reality in itself can be described, then no neutral viewpoint can be adopted to say that one of domain has ontic priority over the other. As a consequence, the ontic priority of the physical world turns out to be a mere metaphysical prejudice: concepts such as bonding, molecular shape and orbital refer to entities belonging to the ontic domain of molecular chemistry, which only depends on its own body of theoretical knowledge. Chemical entities do not owe their existence to an ontically more fundamental level of reality, but to the fact that they are described by theories whose immense predictive and creative power cannot be ignored.

When ontic pluralism is taken seriously, it is possible to claim that the term 'molecule' does not refer to the same ontic item in quantum mechanics and in molecular chemistry. In the quantum theoretical context, a molecule is a quantum system with no shape and no individual differentiated components; even its own nature cannot be conceptualized in terms of the category of individual. A chemical molecule, on the contrary, is an individual object with individual components, whose spatial disposition defines the geometric shape of the molecule. With respect to energy, in the quantum molecule we compute the energy levels of the molecular system as a whole, and it is assumed that those levels are what the imaging of molecular spectrum reveals. In the chemical molecule, by contrast, we compute the energies of the electrons belonging to the molecule, and with them the image of the molecular spectrum is obtained. And the fact that the two results approximately agree in many cases does not cancel the difference between the two ontic pictures. In other words, the word 'molecule' has different meanings in the two domains, the quantum and the chemical, and asking which is the "true" one is as senseless as trying to scrutinize God's mind by means of science.

\section{Acknowledgements:}

This article was made possible through the support of Grant 57919 from the John Templeton Foundation and Grant PICT-2014-2812 from the National Agency of Scientific and Technological Promotion of Argentina.

\section{References}

Anderson, P. W. (1972). "More is different.” Science, 177: 393-396.

Bacciagaluppi, G. (2012). "The role of decoherence in quantum mechanics." In E. N. Zalta (ed.), The Stanford Encyclopedia of Philosophy (Winter 2012 Edition), URL = http://plato.stanford.edu/ archives/ win2012/entries/qm-decoherence/.

Bader, R. (1991). "A quantum theory of molecular structure and its applications." Chemical Reviews, 91: 893-928. 
Bader, R. (1994). Atoms in Molecules: A Quantum Theory. Oxford: Oxford University Press.

Bader, R. (1994). "Atoms in molecules as non-overlapping, bounded, space-filling open quantum system." Foundations of Chemistry, 15: 253-276.

Berlin, Y. A., Burin, A. L., and Goldanskii, V. V. (1996). "The Hund paradox and stabilization of molecular chiral states." Zeitschrift für Physik D, 37: 333-339.

Bernoulli, J. (1713). Ars Conjectandi, Opus Posthumum. Accedit Tractatus de Seriebus Infinitis, et Epistola Gallice Scripta de Ludo Pilae Reticularis. Basel: Thurneysen.

Córdoba, M. and Lombardi, O. (2013). "A Kantian perspective for the philosophy of chemistry." In J.-P. Llored (ed.), The Philosophy of Chemistry: Practices, Methodologies, and Concepts. Cambridge: Cambridge Scholars Publishing.

Cunningham, B. (2001). “The reemergence of 'emergence'.” Philosophy of Science, 68: S62S75.

Dirac, P. A. M. (1929). "Quantum mechanics of many-electron systems." Proceedings of the Royal Society A, 338: 714-733.

Dupré, J. (1993). The Disorder of Things: Metaphysical Foundations of the Disunity of Science. Cambridge MA: Harvard University Press.

Dupré, J. (2000). "Natural kinds." In W. H. Newton-Smith (ed.), A Companion to the Philosophy of Science. Oxford: Blackwell.

Fortin, S., Lombardi, O., and Martínez González, J. C. (2016). "Isomerism and decoherence." Foundations of Chemistry, 18: 225-240.

French, S. and Krause, D. (2006). Identity in Physics: A Historical, Philosophical and Formal Analysis. Oxford: Oxford University Press.

Gross, L., Mohn, F., Moll, N., Liljeroth, P., and Meyer, G. (2009). "The chemical structure of a molecule resolved by atomic force microscopy." Science, 325: 1110-1114.

Hacking, I. (1983). Representing and Intervening. Cambridge: Cambridge University Press.

Hendry, R. F. (2004). "The physicists, the chemists, and the pragmatics of explanation." Philosophy of Science, 71: 1048-59.

Hendry, R. F. (2008). "Two conceptions of the chemical bond." Philosophy of Science, 75 : 909-920.

Hendry, R. F. (2010). “Ontological reduction and molecular structure.” Studies in History and Philosophy of Modern Physics, 41: 183-191.

Hettema, H. (2012). Reducing Chemistry to Physics. Limits, Models, Consequences. Groningen: Rijksuniversiteit Groningen.

Humphreys, P. (2008). "Synchronic and diachronic emergence.” Minds \& Machines, 18: 431442.

Hund. F. (1927). “Zur Deutung der Molekelspektren. III.” Zeitschrift für Physik, 43: 805-826.

Kant, I. (1781) [1998], in P. Guyer and A. Wood (eds.), Critique of Pure Reason, Cambridge: Cambridge University Press.

Kant, I. (1902- ). Gesammelte Schriften. Berlin: Herausgegeben von der Preußischen Akademie der Wissenschaften (Bde. 1-22), der Deutschen Akademie der Wissenschaften zu Berlin (Bd. 23), und der Akademie der Wissenschaften zu Göttingen (Bde. 24, 25, 27-29). 
Kim, J. (2006). "Emergence: core ideas and issues.” Synthese, 151: 547-559.

Kochen, S. and Specker, E. (1967). "The problem of hidden variables in quantum mechanics." Journal of Mathematics and Mechanics, 17: 59-87.

Kuhn, T. (1990), "Dubbing and redubbing: the vulnerability of rigid designation." In W. Savage (ed.), Scientific Theories. Minnesota Studies in the Philosophy of Science, Vol. XIV. Minneapolis: University of Minnesota Press.

Labarca, M. and Lombardi, O. (2010). "Why orbitals do not exist?" Foundations of Chemistry, 12: 149-157.

Lombardi, O. (2014a). "Linking chemistry with physics: arguments and counterarguments." Foundations of Chemistry, 16: 181-192.

Lombardi, O. (2014b). "The ontological autonomy of the chemical world: facing the criticisms." In E. Scerri and L. McIntyre (eds.), Philosophy of Chemistry: Growth of a New Discipline (Boston Studies in the Philosophy and History of Science). Dordrecht: Springer.

Lombardi, O. and Castagnino, M. (2010). "Matters are not so clear on the physical side." Foundations of Chemistry, 12: 159-166.

Lombardi, O. and Labarca, M. (2005). "The ontological autonomy of the chemical world." Foundations of Chemistry 7: 125-148.

Lombardi, O. and Labarca, M. (2006). "The ontological autonomy of the chemical world: A response to Needham." Foundations of Chemistry 8: 81-92.

Lombardi, O. and Labarca, M. (2011). "On the autonomous existence of chemical entities." Current Physical Chemistry 1: 69-75.

Manafu, A. (2011). Emergence and Reduction in Science. A Case Study. PhD Thesis. School of Graduate and Postdoctoral Studies, University of Western Ontario.

O’Connor, T. (1994). “Emergent properties.” American Philosophical Quarterly, 31: 91-104.

Primas, H. (1983). Chemistry, Quantum Mechanics and Reductionism. Berlin: Springer.

Primas, H. (1998). "Emergence in exact natural sciences." Acta Polytechnica Scandinavica, 91: 83-98.

Primas, H. (1994). "Hierarchic quantum descriptions and their associated ontologies." In K. V. Laurikainen, C. Montonen and K. Sunnarborg (eds.), Symposium on the Foundations of Modern Physics 1994. Gif-sur-Yvette: Editions Frontières.

Putnam, H. (1981). Reason, Truth and History. Cambridge: Cambridge University Press.

Quine, W. V. O. (1948). "On what there is.” Review of Metaphysics, 2: 21-38.

Quine, W. V. O. (1981). Theories and Things. Cambridge, MA: The Belknap Pess.

Rohrlich, F. (1988). "Pluralistic ontology and theory reduction in the physical sciences." The British Journal for the Philosophy of Science, 39: 295-312.

Rueger, A. (2000). "Physical emergence, diachronic and synchronic.” Synthese, 124: 297-322.

Scerri, E. R. (2011). “Editorial 37.” Foundations of Chemistry, 13: 1-7.

Scerri, E. R. (2013). "Philosophy of chemistry: where has it been and where is it going." In J.P. Llored (ed.), The Philosophy of Chemistry: Practices, Methodologies, and Concepts. Newcastle: Cambridge Scholars Publishing. 
Schummer, J. (1998). "The chemical core of chemistry I: A conceptual approach.” Hyle, 4: 129-162.

Sutcliffe, B. T. and Woolley, R. G. (2011). "A comment on Editorial 37." Foundations of Chemistry, 13: 93-95.

Sutcliffe, B. T. and Woolley, R. G. (2012). "Atoms and molecules in classical chemistry and quantum mechanics." In R. F. Hendry and A Woody (eds), Handbook of Philosophy of Science. Vol. 6, Philosophy of Chemistry. Oxford: Elsevier.

Teller, P. (1998). "Quantum mechanics and haecceities." In E. Castellani (ed.), Interpreting Bodies. Classical and Quantum Objects in Modern Physics. Princeton: Princeton University Press.

Torretti, R. (2000). "Scientific realism and scientific practice.” In E. Agazzi y M. Pauri (eds.), The Reality of the Unobservable: Observability, Unobservability and their Impact on the Issue of Scientific Realism. Dordrecht: Kluwer.

Torretti, R. (2008). “Objectivity: a Kantian perspective.” In M. Massimi (ed.), Kant and Philosophy of Science Today. Cambridge: Cambridge University Press.

Trost, J. and Hornberger, K. (2009). "Hund's paradox and the collisional stabilization of chiral molecules." Physical Review Letters, 103: 023202.

van Brakel, J. (2000). “The nature of chemical substances.” In N. Bhushan and S. Rosenfeld (eds.), Of Minds and Molecules. New Philosophical Perspectives on Chemistry. New York: Oxford University Press.

Wolff, Ch. (1728). Philosophia Rationalis Sive Logica. Reprint of the 1740 edition with introduction, notes and index by Jean École (ed.). New York: Georg Olms, 1980.

Woolley, R. G. (1978). "Must a molecule have a shape?" Journal of the American Chemical Society, 100: 1073-1078.

Wolley, R. G. (1998). "Is there a quantum definition of a molecule?" Journal of Mathematical Chemistry, 23: 3-12.

Zurek, W. H. (1991). "Decoherence and the transition from quantum to classical." Physics Today, 44: 36-44

Zurek, W. H. (2003). "Decoherence, einselection, and the quantum origins of the classical." Reviews of Modern Physics, 75: 715-776. 